\title{
Immunostaining for thyroid transcription factor 1 , Napsin A, p40, and cytokeratin 5 aids in differential diagnosis of non-small cell lung carcinoma
}

\author{
SATOSHI IKEDA ${ }^{1}$, KEISHI NARUSE ${ }^{2}$, CHIGUSA NAGATA ${ }^{1}$, MASAMI KURAMOCHI ${ }^{3}$, \\ TAKUYA ONUKI $^{3}$, MASAHARU INAGAKI ${ }^{3}$ and KEIKO SUZUKI ${ }^{1}$ \\ ${ }^{1}$ Department of Pathology, Tsuchiura Kyodo General Hospital, Tsuchiura, Ibaraki 300-0053; \\ ${ }^{2}$ Department of Pathology, National Hospital Organization National Kochi Hospital, Kochi 780-8077; \\ ${ }^{3}$ Department of Respiratory Surgery, Tsuchiura Kyodo General Hospital, Tsuchiura, Ibaraki 300-0053, Japan
}

Received July 7, 2014; Accepted February 10, 2015

DOI: $10.3892 / \mathrm{ol} .2015 .3045$

\begin{abstract}
The present study aimed to examine the effectiveness of immunostaining of cytological specimens in discerning squamous from non-squamous cell carcinoma. Various combinations of six antibodies were examined in order to determine the optimal combination for use in differential diagnosis. Immunostaining was performed using tumor imprint smears obtained from 70 lung carcinoma cases. The results were scored based on positive area and intensity. For objective evaluation, scores for thyroid transcription factor 1 (TTF-1), napsin A, and CK CAM5.2 were expressed with positive values, while those for p40, cytokeratin (CK) 5/6 and CK5 were expressed with negative values. Histograms were produced to evaluate which combination was the most effective in differentiating squamous cell carcinoma from adenocarcinoma. The sensitivity for these molecules in adenocarcinoma was $88 \%$ for TTF-1, 85\% for napsin A, and 100\% for CAM5.2. In squamous cell carcinomas, sensitivity was determined to be $90 \%$ for $\mathrm{p} 40,86 \%$ for CK 5 , and $76 \%$ for CK $5 / 6$. The specificity for these molecules was calculated as $100 \%, 95 \%, 43 \%, 98 \%$, $100 \%$ and $95 \%$, respectively. Each combination was evaluated for scoring and the values were averaged. The most effective combination for mode and mean was TTF-1, napsin A, p40, and CK5, for which all adenocarcinomas had a score $>1$, and all squamous cell carcinomas scored $<-2$. Immunostaining scoring may therefore be useful for the differential diagnosis of these carcinomas when a limited number of tumor cells are present.
\end{abstract}

Correspondence to: Mr. Satoshi Ikeda, Department of Pathology, Tsuchiura Kyodo General Hospital, 11-7 Manabe-shinmachi, Tsuchiura, Ibaraki 300-0053, Japan

E-mail: isohama2587@proof.ocn.ne.jp

Key words: immunocy tochemistry, lung cytopathology, scoring

\section{Introduction}

Mortality due to lung cancer accounts for $17 \%$ of all cancer deaths, and worldwide, there are $\sim 1,300,000$ mortalities per year due to this disorder. In 2005 in Japan, lung cancer was responsible for $19 \%$ of all cancer mortalities. The 5-year survival rate for lung cancer is $\sim 30 \%$. In total, $80 \%$ of lung cancer cases are non-small cell carcinoma; this can be divided into non-squamous cell carcinoma and squamous cell carcinoma. Determining a differential diagnosis between non-squamous and squamous cell carcinoma is necessary for effective pulmonary cancer therapy (1). For example, administration of bevacizumab is contraindicated in patients with squamous cell carcinoma due to the possibility of massive bleeding from the lungs, while pemetrexed is only administered for non-squamous cell carcinoma cases, as it only active against non-squamous cell carcinoma (2). Therefore, in patients with lung cancer, precise discrimination between squamous and non-squamous cell carcinoma is vital. However, precise judgment with a limited number of tumor cells obtained from a lung biopsy specimen or in cytology smears is challenging. With biopsy specimens, accurate diagnosis has been demonstrated with the use of immunostaining by a number of studies (3), however, diagnosis based on morphological findings of pap smears is performed in the majority of laboratories. Furthermore, objective methods have been not established for differential diagnosis.

With immunostaining, no consensus has been reached with regard to the use of antibodies, and a companion diagnostic reagent for determining adaptation is essential for molecular-targeted medicines (4). For example, it is necessary to investigate overexpression of the HER-2 protein by immunostaining for adaptation of trastuzumab for mammary or gastric cancer (5). In such cases, the modality and directions for use of antibodies are strictly prescribed (6). Additionally, in lung cancer, an objective method is required for drug selection (2). Therefore, it is hypothesized that the use of immunostaining for differentiation is essential when tumor samples are limited (3). In the present study, immunostaining of cytological specimens was evaluated for its ability to clearly 
Table I. List of antibodies used.

\begin{tabular}{llllrl}
\hline Antigen & Clone & Catalog no. & \multicolumn{1}{c}{ Supplier } & Dilution & Pretreatment \\
\hline TTF-1 & SPT24 & NCL-TTF1 & Leica Microsystems & $150 \mathrm{X}$ & Microwave in pH 6.0 buffer \\
CAM5.2 & CAM5.2 & 349205 & Becton Dickinson $^{\mathrm{a}}$ & $1 \mathrm{X}$ & Microwave in pH 6.0 buffer \\
Napsin A & TMU-Ad02 & 10221 & IBL $^{\mathrm{b}}$ & $50 \mathrm{X}$ & Microwave in pH 6.0 buffer \\
p40 & poly & 418101 & Nichirei Bio-science $^{\mathrm{a}}$ & $1 \mathrm{X}$ & Microwave in pH 6.0 buffer \\
CK5 & XM26 & NCL-L-CK5 & Leica Microsystems $^{\mathrm{a}}$ & $100 \mathrm{X}$ & Microwave in pH 6.0 buffer \\
CK5/6 & D5/16B4 & M7237 & DAKO $^{\mathrm{a}}$ & $75 \mathrm{X}$ & Autoclave in pH 9.0 buffer \\
\hline
\end{tabular}

pH 9.0 pretreatment buffer was purchased from Nichirei Bio-science. ${ }^{a}$ Tokyo, Japan; ' ${ }^{6}$ Takasaki, Gunma, Japan. TTF-1, thyroid transcription factor 1 ; CK, cytokeratin.

distinguish squamous cell carcinoma from adenocarcinoma. For the present objective evaluation, scores for thyroid transcription factor 1 (TTF-1), napsin A, and CK CAM5.2, adenocarcinoma markers were expressed with positive values, while those for p40, cytokeratin (CK) 5/6, and CK5, squamous cell carcinoma markers, were expressed with negative values. This was performed for the purpose of dividing the subjects into two groups by immunostaining. A summed score was determined for each case, and histograms were produced to assess which combination was the most effective in differentiating squamous cell carcinoma from adenocarcinoma.

\section{Materials and methods}

Samples. Consecutive tumor imprint smears were collected from 70 patients between 2011 and 2012 (49 male, 21 females; mean age, 70 years; age range, 51-86 years) with non-small cell carcinoma of the lung, who had undergone resection at Tsuchiura Kyodo General Hospital (Tsuchiura, Japan) from 2011-2012. These were used consecutively without exception. Histological findings revealed that the samples comprised 40 adenocarcinomas, 21 squamous cell carcinomas and 9 samples from other types of non-small cell carcinoma (4 large cell carcinomas, 1 pleomorphic carcinoma and 4 adenosquamous carcinomas). The study was approved by the ethics committee of Tsuchiura Kyodo General Hospital and written informed consent was obtained from the patient or the patient's family.

Immunostaining. Antibodies aganist TTF-1, CK CAM5.2, napsin A, p40, CK5/6 and CK5 were used (Table I). Immunostaining was performed using a Novolink Polymer Detection System (Leica Microsystems; Tokyo Japan) following the appropriate pretreatment as listed in Table I. All procedures were performed as according to the manufacturer's instructions, and the primary antibodies were incubated with the samples at room temperature for $3 \mathrm{~h}$.

Scoring for differential diagnosis. The results of the immunostaining were scored based on positive area and intensity. The expression level was semi-quantitatively evaluated using a combination of proportion and intensity scores from $0-3$ as follows: Score 0 , negative; score 1 , slightly positive, $<10 \%$; score 2 , intermediate, $\geq 10 \%$ and $\leq 90 \%$; score 3 , strongly positive, $>90 \%$ cells were positively stained. The scores for
TTF-1, napsin A, and CAM5.2 were expressed with positive values, while those for $\mathrm{p} 40, \mathrm{CK} 5 / 6$, and CK5 were expressed with negative values. The score for each case was summed and evaluated. For example, when a case of adenocarcinoma was scored as TTF-1 $=2$, CAM5.2 $=3, \mathrm{p} 40=0$ and CK5 $=0$, the total score would be 5 . The various combinations of CAM5.2, napsin A, CK5/6, and CK5 were compared with regard to which was the most effective for determining an accurate diagnosis. Histograms were produced from all cases, and the mean and median scores were calculated to assess which combination was able to most effectively differentiate squamous cell carcinoma from adenocarcinoma. To determine sensitivity and specificity, a score of $>1$ was considered to be positive for adenocarcinoma markers, and a score of $<-1$ was considered to be positive for squamous cell carcinoma markers.

Validation with bronchial lavage fluid. The ability of each combination to precisely differentiate between adenocarcinoma and squamous cell carcinoma was examined using preoperative bronchial lavage fluid samples from 19 cases. In each case, the surgery was performed following bronchial lavage cytology, and a final histological diagnosis was determined. Smears were produced from the remaining bronchial lavage fluid, and immunostaining and scoring were conducted as described previously.

\section{Results}

Immunostaining revealed that TTF-1 and p40 reacted with the tumor cell nuclei, while the remaining antibodies reacted with the cytoplasm (Fig. 1A and B). Napsin A reacted strongly to histiocytes in addition to tumor cells, leading to confusion in the results. Immunostaining results for sensitivity in adenocarcinomas were $88 \%$ for TTF-1, $85 \%$ for napsin A, and $100 \%$ for CAM5.2. In squamous cell carcinomas, sensitivity was $90 \%$ for $\mathrm{p} 40,86 \%$ for CK5, and $76 \%$ for CK5/6. Specificity for each of these molecules was determined to be $100 \%, 95 \%$, $43 \%, 98 \%, 100 \%$ and $95 \%$ respectively. A total of four different combinations, using either CAM5.2 or napsin A, and either CK5/6 or CK5, were assessed, and the mean and median were calculated in order to determine which combination provided the most precise differentiation between the tissue types (Table II). The combination that exhibited the greatest difference between adenocarcinoma and squamous cell 
A
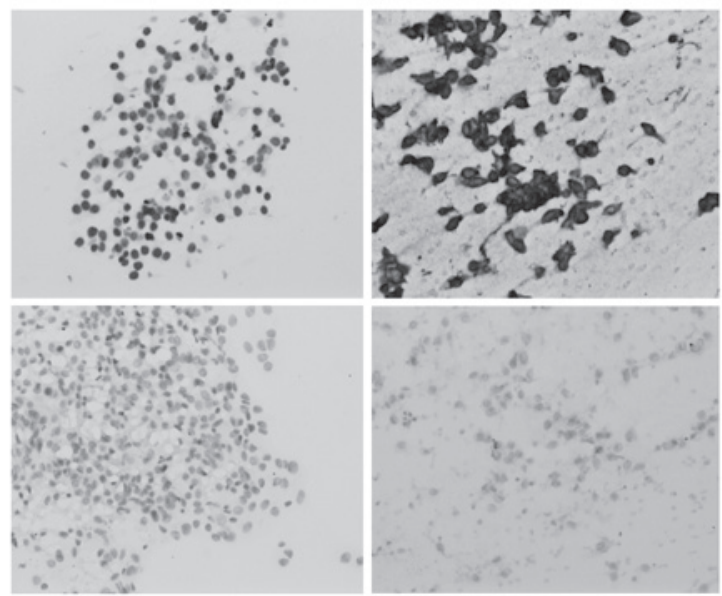

B
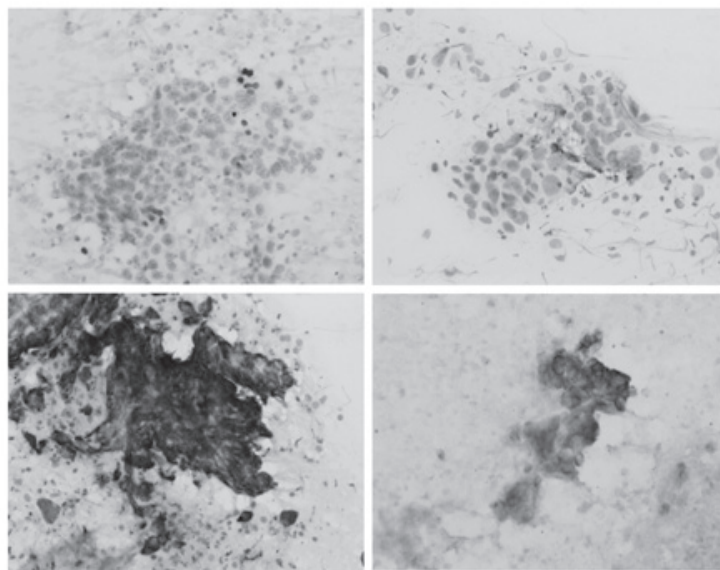
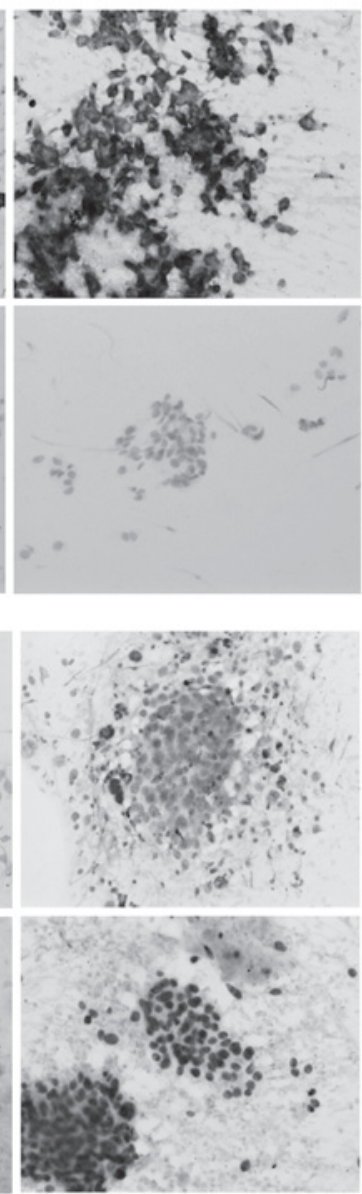

Figure 1. Expression of each protein in (A) adenocarcinoma and (B) squamous cell carcinoma. Top row: Left, thyroid transcription factor 1; center, CAM5.2; right, Napsin A. Bottom row: Left, cytokeratin 5; center, cytokeratin 5/6; right, p40.

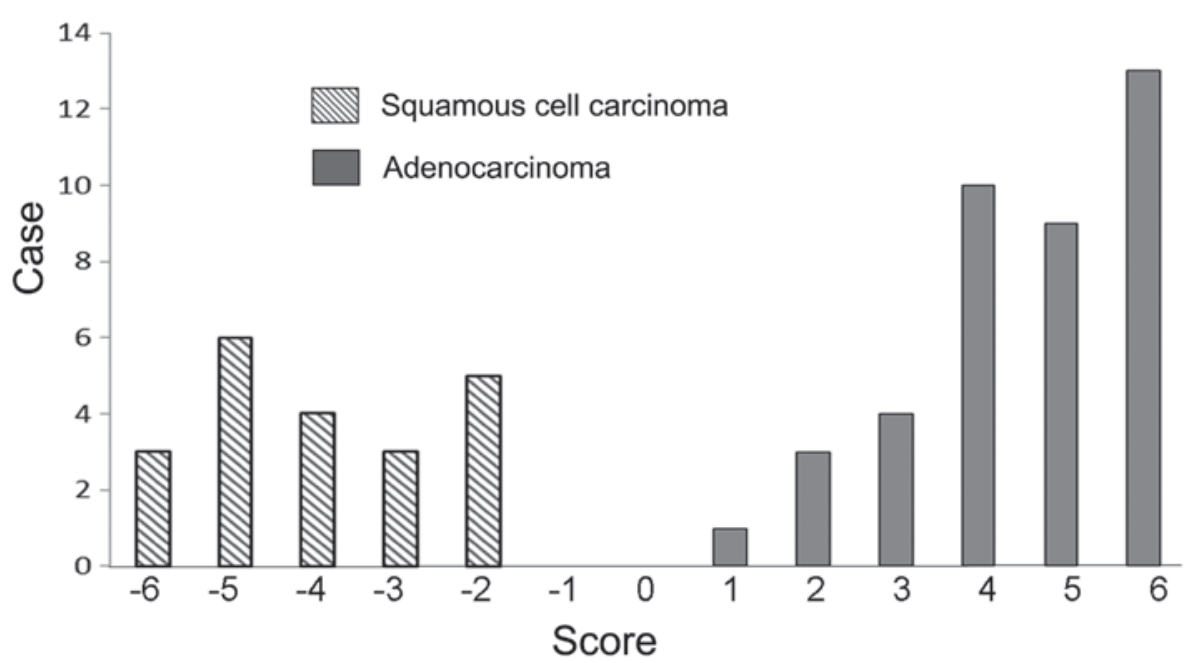

Figure 2. Histograms based on scores using thyroid transcription factor 1, Napsin A, p40, and cytokeratin 5.

carcinoma was TTF-1+napsin A (mean, 4.55) and p40+CK5 (mean, -3.95); this combination was considered to possess the strongest judgment ability (Fig. 2). Using this combination, all adenocarcinomas scored $>1$ and all squamous cell carcinomas scored $<-2$. The majority of cases of other types of non-small cell carcinomas, including large cell carcinoma, were distributed between scores of 2 and -1 (Table III).
The scoring system was subsequently utilized in the same manner using preoperative bronchial lavage fluid samples obtained from 19 cases in order to determine whether this combination was effective. Of the 19 cases, 12 exhibited a score of $>1$ and were determined to be adenocarcinoma, while the remaining 7 cases exhibited scores of $<-2$ and were therefore determined to be squamous cell carcinoma. The 
Table II. Mean and mode scores obtained using each combination of antibodies.

\begin{tabular}{|c|c|c|c|c|c|c|}
\hline \multirow[b]{2}{*}{ Combination } & \multicolumn{2}{|c|}{ Adenocarcinoma } & \multicolumn{2}{|c|}{$\begin{array}{l}\text { Squamous } \\
\text { cell carcinoma }\end{array}$} & \multicolumn{2}{|c|}{$\begin{array}{l}\text { Adenocarcinoma } \\
\text { minus squamous } \\
\text { cell carcinoma }\end{array}$} \\
\hline & Mean & Mode & Mean & Mode & Mean & Mode \\
\hline TTF-1, napsin A, p40, CK5 & 4.55 & 6 & -3.95 & -5 & 8.5 & 11 \\
\hline TTF-1, CAM5.2, p40, CK5/6 & 4.4 & 4 & -3.1 & -3 & 7.5 & 7 \\
\hline TTF-1, CAM5.2, p40, CK5 & 4.88 & 6 & -3.1 & -3 & 7.98 & 9 \\
\hline TTF-1, napsin A, p40, CK5/6 & 4.08 & 4 & -3.91 & -4 & 7.99 & 8 \\
\hline
\end{tabular}

TTF-1, thyroid transcription factor 1 ; CK, cytokeratin.

Table III. Score of large cell carcinomas and adenosquamous carcinomas using antibodies against TTF-1, napsin A, p40 and CK5.

\begin{tabular}{llccccr}
\hline & & \multicolumn{4}{c}{ Score } \\
\cline { 3 - 6 } Case & Histology & TTF-1 & Napsin A & p40 & CK5 & Total \\
\hline 1 & Large cell carcinoma & 0 & 0 & 0 & 1 & -1 \\
2 & Large cell carcinoma & 0 & 0 & 0 & 1 & -1 \\
3 & Large cell carcinoma & 0 & 0 & 0 & 2 & -2 \\
4 & Large cell carcinoma & 0 & 2 & 3 & 0 & -1 \\
5 & Pleomorphic carcinoma & 0 & 1 & 0 & 2 & -1 \\
6 & Adenosquamous carcinoma & 1 & 0 & 0 & 0 & 1 \\
7 & Adenosquamous carcinoma & 1 & 1 & 0 & 1 & 1 \\
8 & Adenosquamous carcinoma & 1 & 2 & 1 & 1 & 1 \\
9 & Adenosquamous carcinoma & 3 & 1 & 0 & 0 & 4 \\
\hline
\end{tabular}

TTF-1, thyroid transcription factor 1 ; CK, cytokeratin.

results were consistent with the final postoperative histological diagnosis in all cases.

\section{Discussion}

In the present study, immunostaining of cytological specimens was assessed with regard to its ability to differentiate squamous cell carcinoma from adenocarcinoma specimens. The differential diagnosis of non-squamous and squamous cell carcinoma is necessary for effective pulmonary cancer therapy specific to the tumor subtype, and a number of studies have been conducted by various institutions with this aim $(3,7)$. Investigations of micro-tissue array sections of lung cancer have shown the value of TTF-1, napsin A, p63 and CK5/6 (3). Yanagita et al (7) reported findings obtained using a cocktail of TTF-1, napsin A, p63, and CK14 antibodies. In this report, the combination of antibodies was useful fordifferential diagnosis; in particular, TTF-1 and napsin A were effective in determining a differential diagnosis. However, to date, no consensus has been reached with regard to the optimum method for the differential diagnosis of lung carcinomas.

TTF-1 is a marker of lung adenocarcinoma and is routinely used for the pathological diagnosis of metastasizing pulmonary adenocarcinoma $(8,9)$. We have previously reported that TTF-1, which reacts in the nucleus, is useful for observations of cytological specimens as the reactions in the nucleus of tumor may identify the cytology of specimen (10).

The expression of napsin A has been reported to be high in lung adenocarcinoma samples (11), while an additional study noted that a large quantity of tissue array samples, with the exception of lung and renal cancer specimens, had extremely low expression (12). Furthermore, the napsin A expression rate was observed to be higher than that of TTF-1 in lung adenocarcinomas. However, the use of napsin A requires particular attention in smears due to its strong expression in histiocytes (13). Shibuki et al (14) also reported that TTF-1 and napsin A were beneficial for improving the diagnostic accuracy of lung adenocarcinoma and squamous cell carcinoma with cytological specimens. In the present study, we distinguish adenocarcinoma cells from histiocytes by reaction of TTF-1 and/or morphological findings.

p63 has generally been used as a marker of squamous cell carcinoma (15), and is considered to be useful and highly sensitive marker for routine pathological diagnosis, as the vast majority of squamous cell carcinoma specimens are positive for this antigen (16). Furthermore, its use is advantageous with 
cytological specimens due to its expression in the nucleus (17). p63 is expressed in poorly differentiated adenocarcinomas, and p40, an isoform of p63, has also been investigated (18). As p40 does not react in the majority of adenocarcinomas, in contrast with $\mathrm{p} 63$, it has been proposed to be a specific marker of squamous cell carcinoma (18).

High-molecular weight CK has also been used as a marker of squamous cell carcinoma, and may be detected by the antibody clone 34ßE12, which recognizes CK subtypes $1,5,10$, and 14 . However, this antibody only reacts in approximately one-third of adenocarcinomas, dependent upon the extent of histological differentiation (10). Thus, antibodies against CK5/6 and CK5, which exhibit no reaction in the vast majority of adenocarcinomas, are currently used for the detection of high-molecular weight CK. In particular, CK5 has been demonstrated to be a reliable marker of triple-negative breast carcinoma (19). In a study of lung cancer, Sethi et al (20) described its utility in diagnosis using cell blocks produced from fine needle aspiration specimens. In this report, both sensitivity and specificity for CK5 for squamous cell carcinoma were $100 \%$.

As our pilot study revealed that the expression of p40 was more specific for squamous cell carcinoma compared with p63, p40 was used in the present experiments (21). The value of TTF-1 as a distinct marker of adenocarcinomas, using immunocytochemistry, has been demonstrated in a number of reports $(10,14)$. In the present study, the expression of p40 and TTF-1 was investigated in conjunction with the expression of CAM5.2, napsin A, CK5/6 and CK5, a combination that has been shown to exhibit effective diagnostic properties $(3,10)$. As the results based on conventional tests of sensitivity and specificity were considered to be ineffective in determining a differential diagnosis, a new method of scoring using objective values was evaluated. The results indicated that the optimal combination comprised the antibodies against TTF-1, napsin A, p40, and CK5. In addition, all six markers were assessed for their diagnostic value when used simultaneously, however, this reduced the judgment ability (data not shown). As the four selected antibodies are utilized in similar antigen retrieval methods, transcription of smears is unnecessary, and double immunostaining is easily conducted. This method demonstrated complete judgment ability in a prospective examination using preoperative bronchial lavage fluid, thus it is proposed to be a beneficial technique for specimens with a limited number of tumor cells.

The four antibodies used in the present study were able to distinguish even poorly differentiated cases of lung carcinoma. However, certain challenges were evident. For example, the score was reduced in cases of mucinous adenocarcinoma that were negative for TTF-1. It may be useful to examine new markers, including mucin $5 \mathrm{~B}$, in this regard (22). In addition, the investigation of specific markers for large cell carcinoma is required. Histological findings and revisions of other methods are necessary to discriminate cases with values between 1 and -2 based on the scoring system developed in the current study.

In conclusion, the present study assessed immunostaining scoring as a means to differentiate between squamous cell carcinoma and adenocarcinoma specimens, in order to determine the most effective combination of antibodies. Antibodies against TTF-1, napsin A, p40, and CK5 provided the best combination. Although numerical values obtained with the present method are unable to differentiate all cases, it may be useful to create an index to aid with diagnosis when a limited number of tumor cells are available, as the majority of adenocarcinoma samples had scores $>2$, and the majority of squamous cell carcinoma samples scored $<-2$.

\section{References}

1. Sandler A, Gray R, Perry MC, et al: Paclitaxel-carboplatin alone or with bevacizumab for non-small-cell lung cancer. N Engl J Med 355: 2542-2550, 2006.

2. Ciuleanu T, Brodowicz T, Zielinski C, et al: Maintenance pemetrexed plus best supportive care versus placebo plus best supportive care for non-small-cell lung cancer: a randomised, double-blind, phase 3 study. Lancet 374: 1432-40, 2009

3. Whithaus K, Fukuoka J, Prihoda TJ and Jagirdar J: Evaluation of napsin A, cytokeratin 5/6, p63, and thyroid transcription factor 1 in adenocarcinoma versus squamous cell carcinoma of the lung. Arch Pathol Lab Med 136: 155-162, 2012.

4. Walker RA, Handy A, Pinder SE, et al: Current issues in diagnostic breast pathology. J Clin Pathol 65: 771-785, 2012.

5. Slamon DJ, Godolphin W, Jones LA, et al: Studies of the HER-2/neu proto-oncogene in human breast and ovarian cancer. Science 244: 707-12, 1989.

6. Pennacchia I, Carbone A, Di Cerbo A, et al: 2013 ASCO/CAP updated guidelines for human epidermal growth factor receptor 2 testing: Impact on routine practice. Breast: Feb 19, 2015 (Epub ahead of print).

7. Yanagita E, Imagawa N, Ohbayashi $\mathrm{C}$ and Itoh $\mathrm{T}$ : Rapid multiplex immunohistochemistry using the 4-antibody cocktail YANA-4 in differentiating primary adenocarcinoma from squamous cell carcinoma of the lung. Appl Immunohistochem Mol Morphol 19: 509-513, 2011.

8. Ng WK, Chow JC and Ng PK: Thyroid transcription factor-1 is highly sensitive and specific in differentiating metastatic pulmonary from extrapulmonary adenocarcinoma in effusion fluid cytology specimens. Cancer 96: 43-48, 2002.

9. Yatabe Y, Mitsudomi T and Takahashi T: TTF-1 expression in pulmonary adenocarcinomas. Am J Surg Pathol 26: 767-773, 2002.

10. Ikeda S, Funakoshi N, Suzuki K, et al: A study of double immunohistochemical staining for differential diagnosis of adenocarcinoma and squamous cell carcinoma in respiratory cytological smears. J Jpn Clin Cytol 42: 5-9, 2003 (In Japanese).

11. Hirano T, Auer G, Maeda M, et al: Human tissue distribution of TA02, which is homologous with a new type of aspartic proteinase, napsin A. Jpn J Cancer Res 91: 1015-1021, 2000.

12. Turner BM, Cagle PT, Sainz IM, et al: Napsin A, a new marker for lung adenocarcinoma, is complementary and more sensitive and specific than thyroid transcription factor 1 in the differential diagnosis of primary pulmonary carcinoma: Evaluation of 1674 cases by tissue microarray. Arch Pathol Lab Med 136: $163-171,2012$.

13. Kakizaki Y, Nakagawa $M$, Honda $T$ and Ishii E: Immunohistochemical study using anti-Napsin A antibody for primary lung adenocarcinoma. J Jpn Clin Cytol 46: 120-121, 2007 (In Japanese).

14. Shibuki Y, Tsuta K, Nomoto K, et al: Immunocytochemical study of specific immunohistochemical markers for primary lung adenocarcinoma: Surfactant apoprotein A, Napsin A, Thyroid transcription factor-1. J Jpn Clin Cytol 45: 6-11, 2006 (In Japanese).

15. Kim DH and Kwon MS: Role of fine needle aspiration cytology, cell block preparation and CD63, P63 and CD56 immunostaining in classifying the specific tumor type of the lung. Acta Cytol 54: 55-59, 2010.

16. Uke M, Rekhi B, Ajit D and Jambhekar NA: The use of p63 as an effective immunomarker in the diagnosis of pulmonary squamous cell carcinomas on de-stained bronchial lavage cytological smears. Cytopathology 21: 56-63, 2010.

17. Aikawa E, Kawahara A, Hattori S, et al: Comparison of the expression levels of napsin A, thyroid transcription factor-1, and p63 in nonsmall cell lung cancer using cytocentrifuged bronchial brushings. Cancer Cytopathol 119: 335-345, 2011.

18. Bishop JA, Teruya-Feldstein J, Westra WH, et al: p40 ( $\Delta$ Np63) is superior to $\mathrm{p} 63$ for the diagnosis of pulmonary squamous cell carcinoma. Mod Pathol 25: 405-415, 2012. 
19. Bhargava R, Beriwal S, McManus K and Dabbs DJ: CK5 is more sensitive than CK5/6 in identifying the 'basal-like' phenotype of breast carcinoma. Am J Clin Pathol 130: 724-730, 2008.

20. Sethi S, Geng L, Shidham VB, et al: Dual color multiplex TTF-1 + Napsin A and p63 + CK5 immunostaining for subcategorizing of poorly differentiated pulmonary non-small carcinomas into adenocarcinoma and squamous cell carcinoma in fine needle aspiration specimens. Cytojournal 9: 10, 2012.
21. Nonaka D. A study of $\Delta \mathrm{Np} 63$ expression in lung non-small cell carcinomas. Am J Surg Pathol 36: 895-9, 2012

22. Li Y, Wang X, Ao M, et al: Aberrant Mucin5B expression in lung adenocarcinomas detected by iTRAQ labeling quantitative proteomics and immunohistochemistry. Clin Proteomics 10: $15,2013$. 